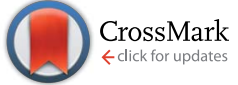

Cite this: RSC Adv., 2017, 7, 12255

Received 13th January 2017

Accepted 15th February 2017

DOI: $10.1039 / \mathrm{c} 7 \mathrm{ra00558j}$

rsc.li/rsc-advances

\section{Cellulose/SnS ${ }_{2}$ composite with enhanced visible- light photocatalytic activity prepared by microwave-assisted ionic liquid method}

\author{
Chunxiang Lin, ${ }^{\text {ab }}$ Moshuqi Zhu, ${ }^{a}$ Teng Zhang, ${ }^{c}$ Yifan Liu, ${ }^{a}$ Yuancai Lv, ${ }^{\text {a }}$ Xiaojuan Li ${ }^{a}$ \\ and Minghua Liu*ab
}

\begin{abstract}
A facile method was used to synthesize cellulose/SnS 2 composites $\left(\mathrm{CE} / \mathrm{SnS} \mathrm{S}_{2}\right)$ by a microwave-assisted ionic liquid (MAIL) method. The effects of ionic liquid types on the structure and properties of $\mathrm{SnS}_{2}$ samples were investigated. Results showed that the ionic liquid played an important role in the control over the morphology, size and photocatalytic performance of the $\mathrm{SnS}_{2}$ particles. The as-synthesized $\mathrm{CE} / \mathrm{SnS}_{2}$ composites were systematically investigated by XRD, SEM, TEM, BET, XPS, UV-visible and PL. It was demonstrated that the obtained $\mathrm{CE} / \mathrm{SnS}_{2}$ composites showed three-dimensional architecture and excellent visible-light photocatalytic activity as photocatalyst for RhB degradation. The superior visiblelight photocatalytic performances for composites were ascribed to their composite structure and the synergistic effects between flower-like $\mathrm{SnS}_{2}$ and cellulose.
\end{abstract}

\section{Introduction}

In the past decades, semiconductor nanomaterials have gained much attention due to their potential applications in solar energy conversion and environmental purification. ${ }^{1-4}$ Among them, tin disulfide $\left(\mathrm{SnS}_{2}\right)$, which has a layered $\mathrm{CdI}_{2}$-type structure with tin atoms sandwiched between two layers of sulfur atoms, ${ }^{5}$ has attracted particular interest in recent years. Compared with the $\mathrm{TiO}_{2}(3.2 \mathrm{eV}), \mathrm{SnS}_{2}$ has a narrower band gap of $\sim 2.2 \mathrm{eV}$, implying more sensitive to visible light. Moreover, it is inexpensive, non-poisonous, and chemically stable, therefore it has proved to be a promising candidate as a photocatalyst for pollutant degradation. ${ }^{6-8}$

It is known that the performance of the nanostructured materials are closely relative to its crystallinity, morphology, size, crystal defect and surface property, which ultimately depend on the preparation methods and preparation conditions. Thus tremendous efforts have been dedicated to the novel methods for the synthesis of high quality nanoparticles with controlled sizes and shapes..$^{9-12}$ To date, a variety of methods have been adopted for the synthesis of well defined $\mathrm{SnS}_{2}$ nanostructures with different morphologies including solvothermal process, hydrothermal process, mechanochemical route, solid state reactions, pyrolysis of organotin precursor, and tetrabutyltin

${ }^{a}$ College of Environment \& Resources, Fuzhou University, Fuzhou 350108, P. R. China. E-mail:mhliu2000@fzu.edu.cn

${ }^{b}$ State Key Laboratory of Photocatalysis on Energy and Environment, College of Chemistry, Fuzhou University, Fuzhou 350002, P. R. China

${ }^{c}$ College of Materials Science and Engineering, Fuzhou University, Fuzhou 350108, China precursor. ${ }^{\mathbf{1 3 - 1 7}}$ However, many of them involved harsh reaction conditions such as high temperature, high pressure or complex preparation procedures. Therefore, it remains a big challenge to develop a simple and facile synthesis which requires only mild reaction conditions under normal atmospheric pressure and room temperature. Most importantly, they can be completely synthesized in a short period of time.

Microwave-assisted ionic liquid (MAIL) method is a novel green chemosynthesis which combines the advantages of microwave (MW) irradiation and room temperature ionic liquids. The particularity of microwave irradiation makes it an effective and attractive method for synthesis of nanomaterials with controllable size and shape.$^{18}$ Compared with conventional heating, it has a more homogeneous heating process and shorter reaction time, generating smaller and more uniform particles. ${ }^{19}$ Many novel nanomaterials with higher photocatalytic activities were prepared by this simple method..$^{20-22}$ On the other hand, ionic liquids (ILs) are drawing increasing attention for the synthesis of inorganic materials owing to their appealing physicochemical properties. ${ }^{23,24}$ Synthesis of photocatalysts in the ILs medium is an exciting and burgeoning area of intense research and has been widely studied recently. It has been reported that the use of ILs as the reaction medium for the synthesis of both pure and modified $\mathrm{TiO}_{2}$ photocatalysts can have a profound effect on the activity, crystallinity and morphology. ${ }^{25-27}$ Research also showed that ILs played an important role to control the size and morphology of the nanomaterials. ${ }^{28,29}$ Additionally, ILs can absorb microwave energy efficiently due to their high polarity. Thus, the combination of ILs and MW irradiation makes MAIL method a useful and rapid way for nanomaterials synthesis with well-defined shapes. ${ }^{30}$ 
In addition, it is of particular interest to immobilize nanoparticles on supporting materials for the improvement of photocatalytic efficiency, because the composite could observably reduce the recombination and enhance the separation rate of photogenerated charge carriers ${ }^{31}$ and own a larger specific surface area, which can benefit the absorption and transportation for pollutant molecules and facilitate the rapid diffusion during the reaction. Of various supports, cellulose can be a suitable binder for immobilization and stabilization of semiconductor nanoparticles due to the significant advantages of cost effectiveness, abundant resources, easier accessibility and possession of a lot of surface hydroxyl groups. ${ }^{32}$

Herein, we report a facile one-pot method, MAIL, for the synthesis of cellulose/ $\mathrm{SnS}_{2}$ composite by taking advantage of the dissolution of cellulose in an ionic liquid, and thereby obtaining a uniform $\mathrm{SnS}_{2}$ coating onto cellulose. The effect of different types of ILs on the phase structure and microstructure of $\mathrm{SnS}_{2}$ were discussed and the photocatalytic activities of the composite were evaluated by degrading rhodamine B (RhB) under visible light.

\section{Experimental}

\subsection{Materials}

A series of 1-alkyl-3-methylimidazolium salts [ $\left.\mathrm{C}_{n} \mathrm{MIM}\right] \mathrm{X}$ ionic liquids $\left(n=4,6 ; \mathrm{X}=\mathrm{BF}_{4}, \mathrm{Br}, \mathrm{Cl}\right.$, Aco; purity $>99 \%$; named $\mathrm{IL}(1)-$ $\mathrm{IL}(5)$, representative $\left[\mathrm{C}_{4} \mathrm{MIM}\right] \mathrm{BF}_{4}, \quad\left[\mathrm{C}_{4} \mathrm{MIM}\right] \mathrm{Br}, \quad\left[\mathrm{C}_{6} \mathrm{MIM}\right] \mathrm{Cl}$, $\left[\mathrm{C}_{4} \mathrm{MIM}\right] \mathrm{Cl}$ and $\left[\mathrm{C}_{4} \mathrm{MIM}\right] \mathrm{AcO}$ respectively) were purchased from Shanghai Chengjie Chemical Co., Ltd, China, and their general structures are shown in Scheme 1. All other reagents used in this research were of analytical grade and utilized as-received without further purification. For reference, the structure of $\mathrm{RhB}$ is shown in Scheme 2.

\subsection{Preparation $\mathrm{CE} / \mathrm{SnS}_{2}$ composite via microwave-assisted IL method}

In a typical procedure, $0.7 \mathrm{~g}$ CE was dissolved in $15 \mathrm{~g} \mathrm{IL}(4)$ to form a transparent solution, and then $3 \mathrm{mmol} \mathrm{SnCl}_{4} \cdot 5 \mathrm{H}_{2} \mathrm{O}$ and $6 \mathrm{mmol}$ thioacetamide were ultrasound dissolved in this $\mathrm{CE} / \mathrm{IL}$ mixture. Then the mixture was sealed in a specialized glass tube under microwave irradiation at $120^{\circ} \mathrm{C}$ for $120 \mathrm{~min}$ (pressure: $\sim 2$ bar) in a single mode microwave reactor (Nova, EU Microwave Chemistry). The as-formed precipitates were collected by centrifuging, copious washing with ethanol and drying.

To investigate the effect of ILs on the structure of $\mathrm{SnS}_{2}$, the pristine $\mathrm{SnS}_{2}$ material was prepared by a similar procedure except for the dissolution of cellulose, the corresponding products were labelled as IL1-IL5, respectively. For comparison,

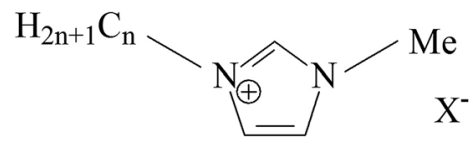

Scheme 1 Molecular structure of the $\left[\mathrm{C}_{n} \mathrm{MIM}\right] \mathrm{X}$ ionic liquids ( $n=4$ or 6; $\mathrm{X}=\mathrm{BF}_{4}, \mathrm{Br}, \mathrm{Cl}$ or $\left.\mathrm{AcO}\right)$.

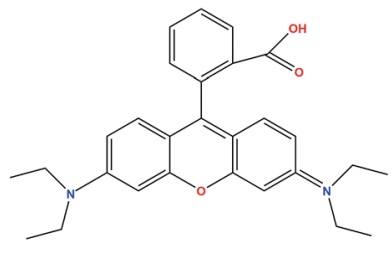

$\mathrm{Cl}^{-1}$

Scheme 2 Molecular structure of the RhB.

this experiment was also done by using glycol instead of ILs as medium, and was labelled as NIL.

\subsection{Materials characterization}

The crystalline phases of $\mathrm{SnS}_{2}$ and $\mathrm{CE} / \mathrm{SnS}_{2}$ composites were evaluated by X-ray diffraction (XRD, MinFlex600, $\mathrm{Cu} \mathrm{K}, \lambda=$ $0.15418 \mathrm{~nm}$ ). Fourier transform infrared (FTIR) spectroscopy was performed using a Nicolet iS10 FT-IR spectrometer by $\mathrm{KBr}$ disk. Thermal stability of samples was examined on a thermogravimetric analyzer (NETZSCH STA449C, Germany) in $\mathrm{N}_{2}$ atmosphere. The morphologies and microstructures of the prepared samples were analysed by field-emission scanning electron microscopy (FE-SEM, Nova NanoSEM 230) equipped with an energy-dispersive X-ray spectroscopy (EDX) system, and transmission electron microscopy (TEM TECNAI G2F20). The surface compositions of the catalyst were evaluated by X-ray photoelectron spectra (XPS, Thermo Scientific ESCALAB 250). $\mathrm{N}_{2}$ adsorption-desorption isotherms were obtained at $-196{ }^{\circ} \mathrm{C}$ on a Micromeritics ASAP 2020 Sorptometer using static adsorption procedures, and the BET surface areas and pore size distributions were calculated by using $\mathrm{N}_{2}$ adsorption-desorption isotherms. UV-vis diffuse reflectance spectroscopy (DRS) was recorded on a Carry 500-Scan spectrophotometer.

The photoluminescence (PL) spectra were recorded at room temperature using a FL/FS 900 spectrophotometer equipped with a $300 \mathrm{~W}$ xenon lamp as the excitation source.

\subsection{Photoelectrochemical measurements and photocatalytic test}

Photocurrents were measured with an electrochemical analyzer (CHI650E) in a standard three-electrode system using the prepared samples as the working electrodes with an active area of $c a .0 .5 \mathrm{~cm}^{2}$, a Pt wire as the counter electrode, and $\mathrm{Ag} / \mathrm{AgCl}$ (saturated $\mathrm{KCl}$ ) as a reference electrode. A $300 \mathrm{~W}$ xenon arc lamp through a UV-cutoff filter $(\leq 420 \mathrm{~nm})$ served as a visiblelight source, and $0.5 \mathrm{M} \mathrm{Na}_{2} \mathrm{SO}_{4}$ solution was used as the electrolyte.

Photocatalytic activity of the samples was evaluated by the photocatalytic degradation of rhodamine $\mathrm{B}\left(\mathrm{RhB}, 20 \mathrm{mg} \mathrm{L}^{-1}\right)$ in an aqueous solution under visible-light irradiation. The photocatalytic system included a $300 \mathrm{~W}$ Xe arc lamp with a UV-cut off filter $(\lambda>420 \mathrm{~nm})$, circulation of water through an external cooling coil and a ventilating fan, which were used to prevent any thermal catalytic effects. All experiments were conducted at room temperature in air. In a typical photocatalytic experiment, 
$0.03 \mathrm{~g}$ photocatalyst was added into $100 \mathrm{ml}$ pollutant solution in a reaction cell with a Pyrex jacket. Prior to irradiation, the suspension was magnetically stirred in the dark for $1 \mathrm{~h}$ to reach an adsorption-desorption equilibrium of the pollutant on the catalyst surface. Then these suspensions were exposed to visible-light irradiation under magnetic stirring. At given time intervals, about $5 \mathrm{ml}$ suspensions were collected and centrifuged (13 $000 \mathrm{rpm}, 15 \mathrm{~min}$ ) to remove the photocatalyst particles. The pollutant concentration of the obtained solution was analyzed by a UV-VIS spectrophotometer (Hitachi, U-3310) by checking the absorbance at $553 \mathrm{~nm}$.

\section{Results and discussion}

\subsection{Effect of ionic liquids on structures and properties of $\mathrm{SnS}_{2}$ samples}

In order to perform the effect of different kinds of ionic liquid on the structures and properties of $\mathrm{SnS}_{2}$ under microwave radiation, pristine $\mathrm{SnS}_{2}$ samples were prepared via MAIL method using IL(1)-IL(5) as medium without cellulose addition.

Fig. 1 shows the XRD patterns of the different samples prepared in IL(1)-IL(5). It can be seen that IL1-IL4 and NIL show peaks at $2 \theta=15.02^{\circ}, 28.25^{\circ}, 32.14^{\circ}, 50.04^{\circ}$, which could be indexed to reflections from (001), (100), (011), (110) planes of pure hexagonal $\mathrm{SnS}_{2}$ and they are also in good agreement with the standard card (PDF\#89-2028). No trace of impurity peaks could be detected in these samples, indicating the phase purity of the products. While IL5 shows amorphous structure, which may be ascribed to the effect of anion in $\operatorname{IL}(5)$. The above results indicate that the completely crystalline and pure-phase $\mathrm{SnS}_{2}$ nanoparticles could be obtained by this facile MAIL route using $\mathrm{IL}(1)-\mathrm{IL}(4)$. Moreover, the intensity of the characteristic diffraction peaks of IL1-IL4 increased in comparison with NIL (sample prepared in glycol).

Ionic liquid also have great influence on the morphology of as-prepared $\mathrm{SnS}_{2}$ samples. The morphologies of all samples were characterized by FE-SEM images, as shown in Fig. 2. When only microwave technique was used (without any IL), a large quantity of nanosheet morphology is observed (Fig. 2a). Depending on the IL used, different shapes are obtained. The

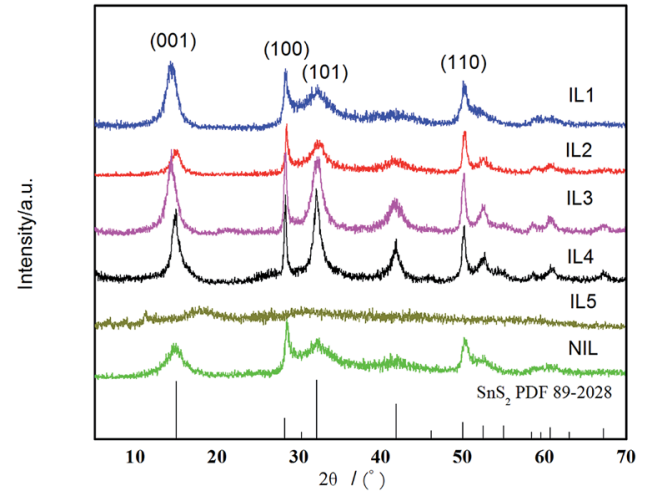

Fig. 1 XRD patterns of $\mathrm{SnS}_{2}$ samples prepared in ILs (IL1-IL5) and glycol (NIL).
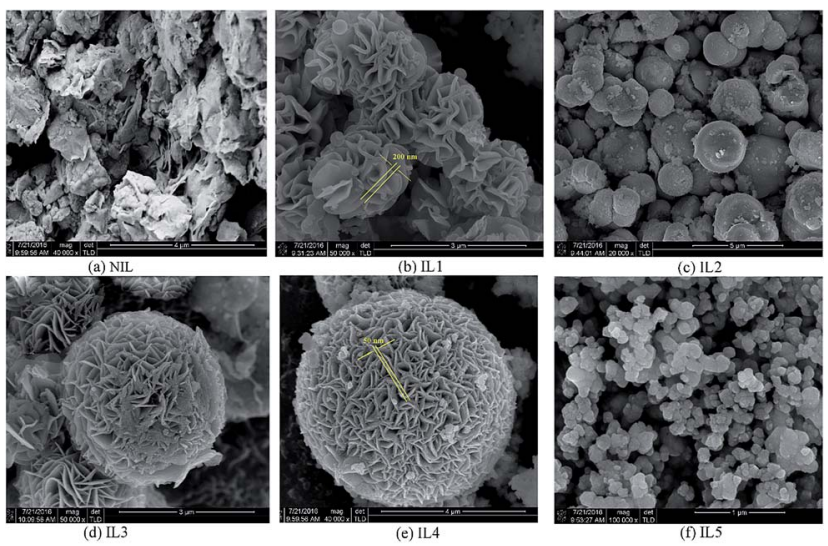

Fig. 2 FE-SEM images of $\mathrm{SnS}_{2}$ samples prepared in ILs (IL1-IL5) and glycol (NIL).

SEM image of IL1 clearly shows carnation flower-like architectures assembled from 2D nanopetals building blocks with thicknesses of 150-200 nm. In IL2 micro-sized spherical-like particles is observed where each sphere is around $2-4 \mu \mathrm{m}$ in diameter. The SEM images of IL3 and IL4 show 3D flowerlike microspheres with different sizes of diameter $(2-3 \mu \mathrm{m}$ for IL3 and 4-6 $\mu \mathrm{m}$ for IL4) that are composed of hundreds of nanosheets with thicknesses of 30-50 nm (Fig. 2d and e). Just like a Red Embroidery Ball. The 2D nanosheets interweave together to form an open porous structure. While the morphology of IL5 presents uniform and reunited nanoparticles with diameter of 100-200 nm. These results reveal that the ILs can play an important role in controlling the growth of $\mathrm{SnS}_{2}$ crystals and formation of uniform morphology. Moreover, the length of alkyl side chain in the cation has no effect on morphology of $\mathrm{SnS}_{2}$ but affect the particle size, while the types of anions in imidazolium ILs have great influence on morphology and shape.

TEM images were performed to further investigate the structure of the as-synthesized $\mathrm{SnS}_{2}$ in different ILs. When no ILs used, it is clear that the irregular nanosheets is made up of numerous uniformly sized nanoparticles (Fig. 3a). While the TEM images of $\mathrm{SnS}_{2}$ presented in Fig. 3b-f reveal that the 3D micro/nano structures prepared by using ILs are assembled by $2 \mathrm{D}$ nanosheets. The TEM results showed in Fig. $3 \mathrm{~b}-\mathrm{f}$ exhibit that the products are flower-like, spherical-like, irregular nanosheets and nanoparticles structures, respectively, similar to the SEM results (Fig. 2b-f).

The specific surface areas and pore sizes of $\mathrm{SnS}_{2}$ samples were determined by nitrogen adsorption-desorption isotherms according to Brunauer-Emmett-Teller (BET). As observed in Fig. 4, the isotherms of all the as-synthesized $\mathrm{SnS}_{2}$ are type IV isotherm with a hysteresis loop according to IUPAC classification, which indicates the presence of mesopores in the products. ${ }^{33}$ The specific surface areas of all samples, calculated by the multi-point Brunauer-Emmett-Teller (BET) method, are listed in Table 1. It is observed that the surface area increases IL2 $<$ NIL $<$ IL1 < IL4 < IL3 < IL5.

The optical property of all $\mathrm{SnS}_{2}$ samples were investigated with UV-vis diffuse reflectance spectrometer (DRS) as shown in 


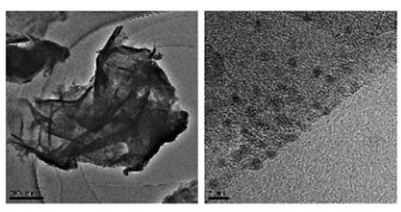

(a) NIL

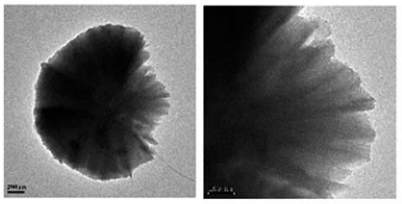

(c) IL2

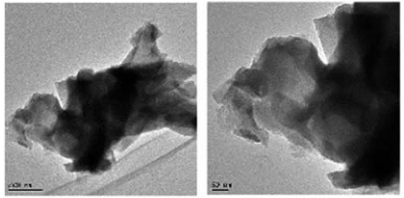

(e) II.4

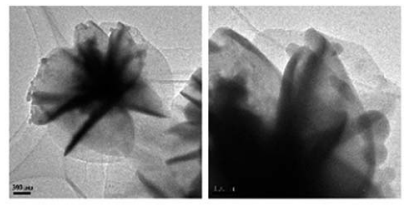

(b) IL1

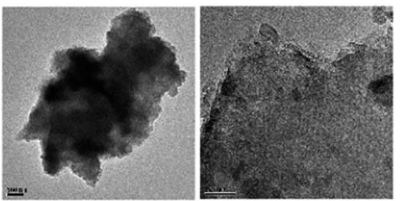

(d) IL3

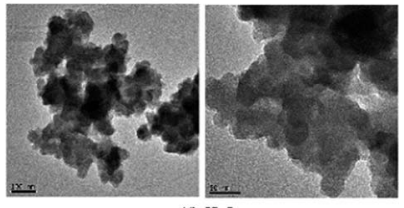

(f) IL5
Fig. 3 TEM images of $\mathrm{SnS}_{2}$ samples prepared in ILs (IL1-IL5) and glycol (NIL).

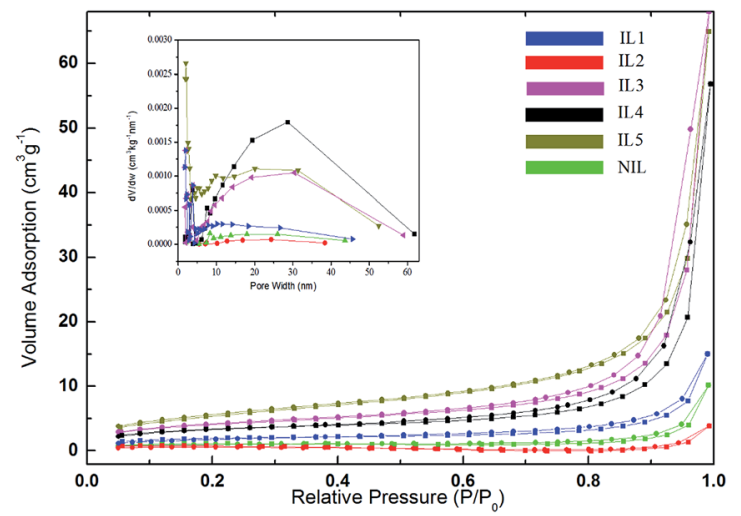

Fig. $4 \quad \mathrm{~N}_{2}$ adsorption-desorption isotherm of $\mathrm{SnS}_{2}$ samples prepared in ILs (IL1-IL5) and glycol (NIL).

Fig. 5a, from which we see that the IL1-4 exhibit much higher light absorption intensity than NIL and IL5, which may be ascribe to the higher crystalline of IL1-IL4. The optical band gap energy can be calculated by the following formula:

$$
\alpha h \nu=A\left(h \nu-E_{\mathrm{g}}\right)^{1 / 2}
$$

where $\alpha, h, \nu, E_{\mathrm{g}}$ and $A$ represent the absorption coefficient, Planck constant, the light frequency, the band gap, and a constant, respectively. Therefore, the band gap energies of these samples can be estimated from plot $(\alpha h \nu)^{2}$ versus photon

Table 1 Specific surface areas of $\mathrm{SnS}_{2}$ samples prepared in NIL and IL(1) - (5)

\begin{tabular}{lllllll}
\hline ILs & NIL & IL1 & IL2 & IL3 & IL4 & IL5 \\
\hline
\end{tabular}

$\begin{array}{lllllll}\text { Specific surface areas } & 3.34 & 6.5060 & 1.89 & 16.6950 & 14.4730 & 23.1462\end{array}$

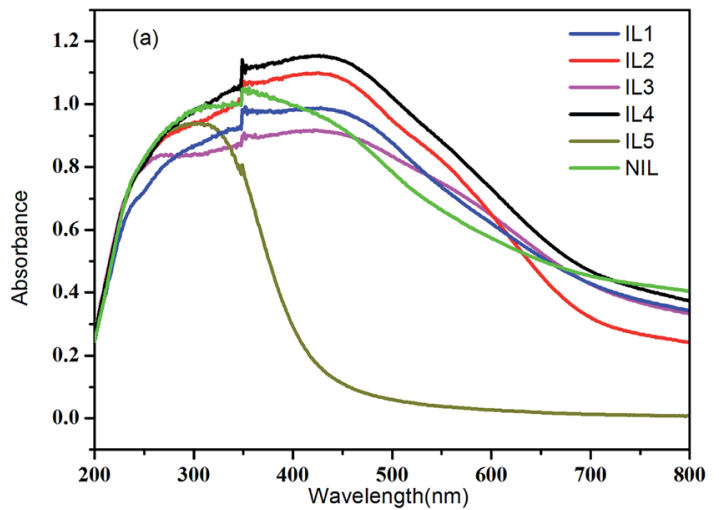

(a)

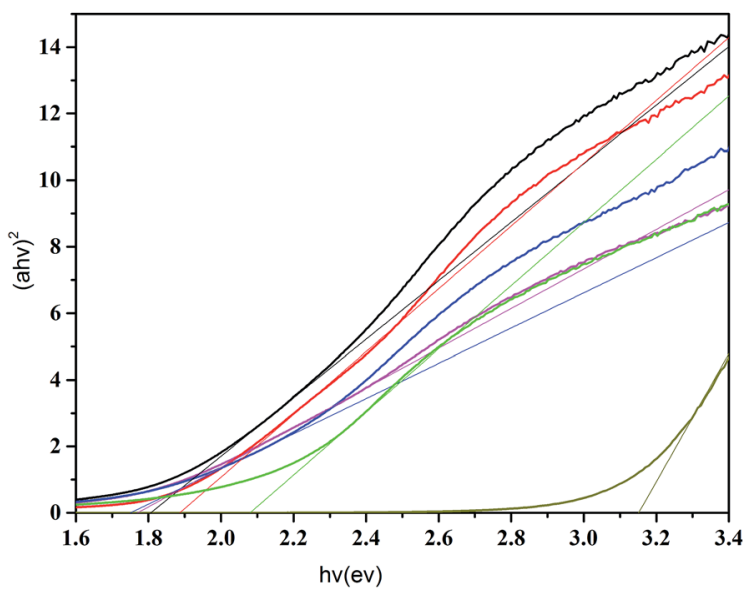

(b)

Fig. 5 UV-vis solid absorption spectra of $\mathrm{SnS}_{2}$ samples (a) and the corresponding band gap values (b).

energy $(h \nu)$. The intercept of the tangent to the $X$ axis could provide a good approximation of the band gap energy. As shown in Fig. 5b, the derived band gaps are estimated to be $1.76 \mathrm{eV}$, $1.89 \mathrm{eV}, 1.78 \mathrm{eV}, 1.80 \mathrm{eV}, 3.15 \mathrm{eV}$ and $2.06 \mathrm{eV}$ for IL1-5 and NIL, respectively. It is clearly seen that IL1, IL3 and IL4 has the smaller band gap in comparison with other samples, and IL5 has the largest value. Combined with the SEM images, we find that flower-like samples (IL1, IL3 and IL4) display strong absorption in the visible light region mainly due to its perfect light-trapping structure, which may lead to an increase in the generation of electron-hole pairs and then enhances the photocatalytic activity of the $\mathrm{SnS}_{2}$ samples. ${ }^{34-36}$

The above results confirm that the size, morphology and structure of the nanomaterials can influence the optical properties greatly, thus offer an effective way of tuning the band gap. $^{37}$

To examine the photocatalytic activities of the all $\mathrm{SnS}_{2}$ samples, the degradation of aqueous solutions of RhB $(20 \mathrm{mg}$ $\left.\mathrm{L}^{-1}\right)$ under visible light $(\lambda>420 \mathrm{~nm})$ irradiation were performed. A blank test (RhB without any catalyst) exhibits no degradation under visible light irradiation, which indicates the photolysis of $\mathrm{RhB}$ could be ignored. It can be seen from the curves of RhB absorption rates (Fig. 6) that the adsorption-desorption 


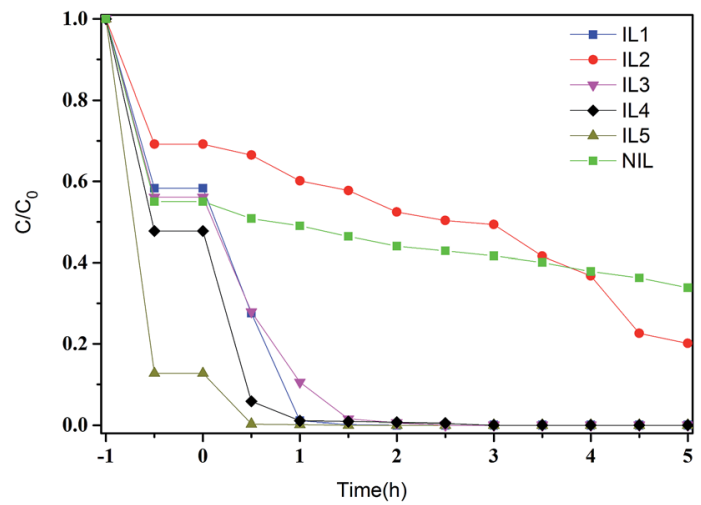

Fig. 6 Photocatalytic activities for degradation of RhB dye under visible-light irradiation in the presence of $\mathrm{SnS}_{2}$ samples.

equilibrium of RhB on the all samples have been achieved in $60 \mathrm{~min}$ before visible-light irradiation. As can be seen from Fig. 6, IL5 possesses strong adsorption ability towards RhB with $87.3 \%$ of adsorptive ratios before visible light irradiation. That may be due to the larger specific surface area of IL5. The other samples show different photocatalytic activities towards RhB whose photocatalytic activity can be judged roughly by the slope of the degradation curves, which decrease IL4 > IL1 > IL3 > NIL > IL2. It can be presented that IL1, IL3 and IL4 show higher photocatalytic activity than other samples, the possible reason may be ascribed to the combination of smaller band gap energy and the larger specific surface area compared with other samples.

Based on the above results, it is found that although the synthetic conditions are identical for samples prepared in different ILs, $\mathrm{SnS}_{2}$ nano/micro particles show different crystalline, structures, morphologies, size and performance. It can be concluded that the ionic liquid plays an important role in the control over the morphology and size of the product, the reason may be ascribe to different mechanisms, including hydrogen bonds, $\pi-\pi$ stack interactions, self-assembly mechanism, electrostatic attraction, and so on. ${ }^{38,39}$ Meanwhile, it's also presented that the $\mathrm{SnS}_{2}$ samples prepared in imidazole chloride salts, IL(3) and IL(4), exhibit higher photocatalytic performance than other samples.

In the following parts, IL(4), as an efficient solvent for cellulose, was selected as the reaction medium due to the good performance of $\mathrm{SnS}_{2}$ prepared in it; we will discuss the effects of cellulose support on the structures, morphologies and photocatalytic properties of final products using IL(4) as reaction medium under microwave irradiation.

\section{2 $\mathrm{CE} / \mathrm{SnS}_{2}$ composites synthesis and characterization}

3.2.1 Characterization. By using $\mathrm{CE}$ as a supporting matrix, $\mathrm{CE} / \mathrm{SnS}_{2}$ was prepared via MAIL methods. The IL(4) (BMIMCl) was used as the reaction medium. XRD pattern of the assynthesized product in Fig. 7 shows that all the diffraction peaks can be indexed to the $\mathrm{SnS}_{2}$ phase $\left(15.02^{\circ}, 28.25^{\circ}, 32.14^{\circ}\right.$, $50.04^{\circ}$ ), along with a very weak peak at $2 \theta=21.32^{\circ}$, indicating the existence of regenerated CE.

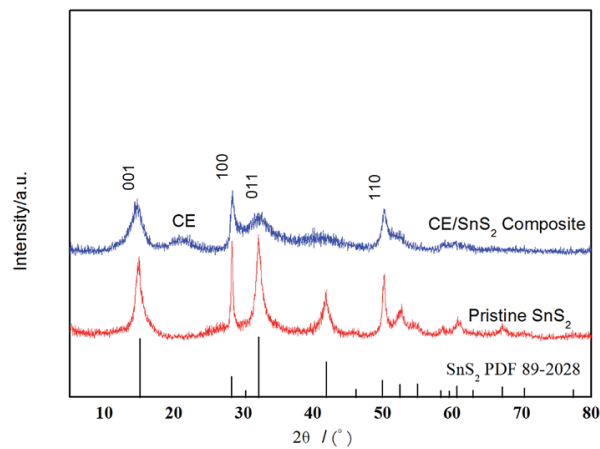

Fig. 7 XRD pattern of pristine $\mathrm{SnS}_{2}$ (IL4) and $\mathrm{CE} / \mathrm{SnS}_{2}$ composite.

The chemical structure of as-synthesized $\mathrm{CE} / \mathrm{SnS} \mathrm{S}_{2}$ composite has been examined using FT-IR spectra, as shown in Fig. 8. It can be seen that the FTIR spectrum of $\mathrm{CE} / \mathrm{SnS}_{2}$ composite is similar with $\mathrm{CE}$ and contains all the characteristic absorption of $\mathrm{CE}$. However, the bands centered at around $3400 \mathrm{~cm}^{-1}$, correspond to $-\mathrm{OH}$ vibration in $\mathrm{CE} / \mathrm{SnS}_{2}$ composite, weakened or shifted due to the interaction between $\mathrm{CE}$ and $\mathrm{SnS}_{2}$. In addition, the weak peak corresponds to $\mathrm{Sn}-\mathrm{S}$ bond that would be appeared at about $630 \mathrm{~cm}^{-1}$ is hard to observe due to overlapping with other peaks.

Thermal gravimetric analysis (TGA) was use to reveal the degradation profiles of as-synthesized products. As shown in Fig. 9, the initial weight losses of three lines below $200{ }^{\circ} \mathrm{C}(5-$ $7 \%$ ) are attributed to some of the adsorbed water in the products. The main peaks corresponding to the thermal degradation of CE (line a) were observed between 270 and $280{ }^{\circ} \mathrm{C}$ and undergoes two stages.

The first weight of loss continues up to $370{ }^{\circ} \mathrm{C}$ during which there is $72 \%$ weight loss. And the second stage starts at $370{ }^{\circ} \mathrm{C}$, and a residue of $10.5 \mathrm{wt} \%$ remains after heating to $650{ }^{\circ} \mathrm{C}$. While the thermal stability of $\mathrm{CE} / \mathrm{SnS}_{2}$ composite presents much higher (line b), which shows an initially decomposition of $18 \%$ mass loss in the temperature range of $270-320{ }^{\circ} \mathrm{C}$, followed by another $13 \%$ mass loss at temperatures between 320 and $750{ }^{\circ} \mathrm{C}$, leaving significantly high (around $62 \%$ ) residue at $750{ }^{\circ} \mathrm{C}$. The improved thermal stability is ascribed to the complex of $\mathrm{SnS}_{2}$ (line c) by MAIL method. Moreover, the TGA analysis can be also employed to determine the amount of CE presents in $\mathrm{CE} / \mathrm{SnS}_{2}$ composite. The weight loss of composites are mainly attributed

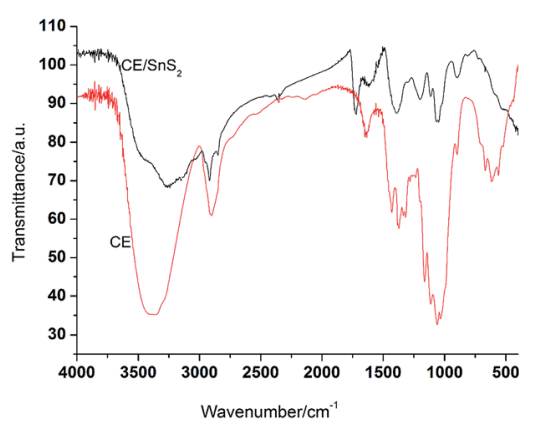

Fig. $8 \mathrm{FTIR}$ spectra of $\mathrm{CE}, \mathrm{CE} / \mathrm{SnS}_{2}$ composite and pristine $\mathrm{SnS}_{2}$. 


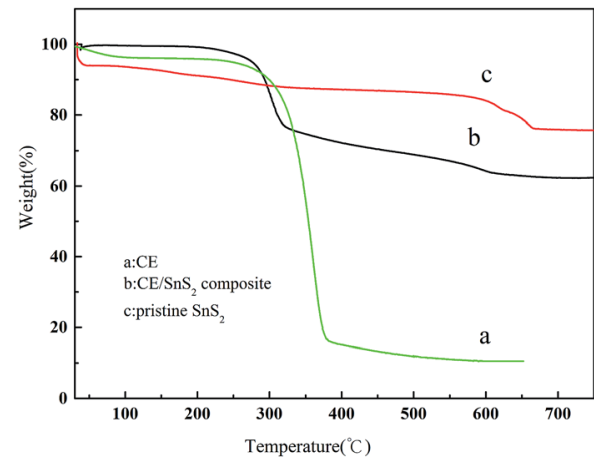

Fig. 9 TGA of $\mathrm{CE}, \mathrm{CE} / \mathrm{SnS} \mathrm{S}_{2}$ composite and pristine $\mathrm{SnS}_{2}$.

to the CE composition and phase transformation from $\mathrm{SnS}_{2}$ to $\mathrm{SnO}_{2}$ (the substitution of $\mathrm{S}$ by $\mathrm{O}$ atoms), which can be calculated from line a and line c, respectively. Thus, the weight fraction of the CE supporter is determined to be $\sim 20 \%$.

The general morphology of $\mathrm{CE} / \mathrm{SnS}_{2}$ composites is shown in Fig. 10. The composites display a 3D flower-like structure composed of $2 \mathrm{D} \mathrm{SnS}_{2}$ nanoplates which are tightly anchored on the surface of CE. Without using CE in the synthetic process, the pristine $\mathrm{SnS}_{2}$ materials prepared in $\mathrm{IL}(4)$ (Fig. 2e) exhibit a characteristic of 3D Red-Embroidery-Ball-like structure with thicker nanosheets than that of the $\mathrm{CE} / \mathrm{SnS}_{2}$ composites, implying that the crystal growth and self-assembly process of $\mathrm{SnS}_{2}$ materials was hindered by the presence of CE.

Also, the scanning transmission electron microscopy (STEM) images of the composite (Fig. 10) and the corresponding elemental mapping images show matched spatial distribution of S, Sn and C, indicating that the $\mathrm{SnS}_{2}$ particles are generated on the surface of CE, in accordance with the SEM observation.

Moreover, energy dispersive X-ray spectroscopy (EDS) analysis (shown in Fig. 11) indicates that the $\mathrm{Sn} / \mathrm{S}$ molar ratio is approximately $1: 2$, further confirming the formation of $\mathrm{SnS}_{2}$ on the CE surface.

Further insight into the morphology and microstructure of $\mathrm{SnS}_{2}$ nanoparticles loaded on CE is displayed in the TEM image in Fig. 12a. It is clear that the as-synthesized composites are built by the intertwining of single crystalline nanosheets. The

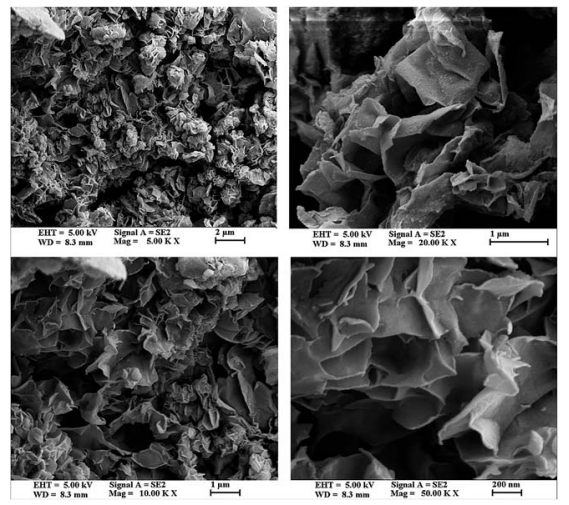

Fig. 10 The morphology of $\mathrm{CE} / \mathrm{SnS}_{2}$ composites.
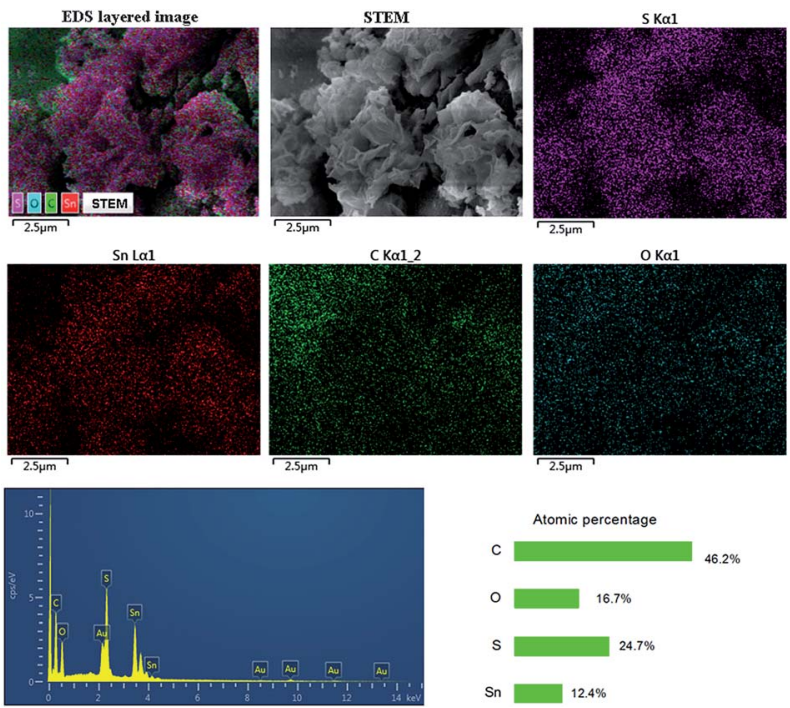

Fig. 11 STEM images of the composite and the corresponding elemental mapping images.

relatively light regions are thin sheets lying along the substrate, while the dark regions mean that nanosheets arrange perpendicular to the substrate or convolute during the reaction process. The HRTEM image in Fig. 12b exhibits parallel fringes with a spacing of $0.52 \mathrm{~nm}$, in good agreement with the interlayer spacing of $\mathrm{SnS}_{2}$.

The surface composition of the $\mathrm{CE} / \mathrm{SnS}_{2}$ composite was analyzed by XPS as shown in Fig. 13. The binding energy values obtained in the XPS analysis were standardized by referencing the C 1s peak to $284.60 \mathrm{eV}$. Fig. 13a is a typical XPS survey spectrum of different atoms, which shows that the $\mathrm{CE} / \mathrm{SnS}_{2}$ composite is composed of $\mathrm{Sn}, \mathrm{S}$ and $\mathrm{C}$. The $\mathrm{C}$ element is ascribed to the cellulose support. Fig. 13b shows the high-resolution XPS spectrum of the Sn 3d. The two strong peaks at around 486.5 and $495.0 \mathrm{eV}$ are assigned to $\mathrm{Sn} 3 \mathrm{~d}_{3 / 2}$ and $3 \mathrm{~d}_{5 / 2},{ }^{40}$ respectively, which is characteristic of $\mathrm{Sn}^{4+}$ in $\mathrm{SnS}_{2}$. The XPS spectrum of $\mathrm{S} 2 \mathrm{p}$ is shown in Fig. 13c. The main peak located at 161.7 and shoulder peak at $162.8 \mathrm{eV}$ can be attributed to $\mathrm{S} 2 \mathrm{p}_{3 / 2}$ and $\mathrm{S} 2 \mathrm{p}_{1 / 2}$, respectively, which is in agreement with the reported data. ${ }^{\mathbf{4 1 , 4 2}}$

The atomic ratio of $\mathrm{Sn} / \mathrm{S}$ calculated by the integral area of $\mathrm{Sn}$ $3 \mathrm{~d}$ to $\mathrm{S} 2 \mathrm{p}$ is about $1: 1.92$, which is close to stoichiometric ratio
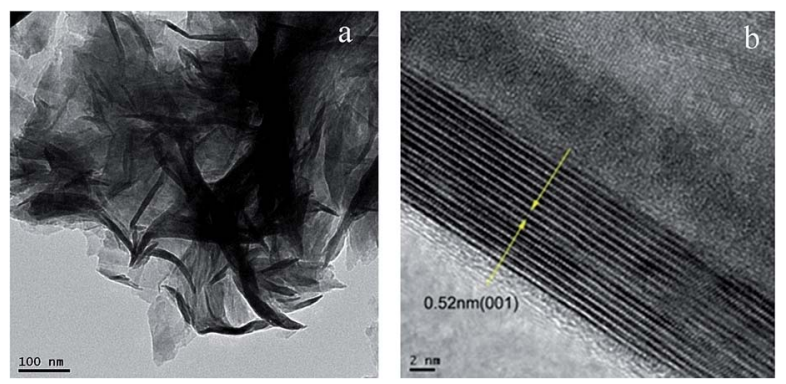

Fig. 12 Representative TEM image (a) and HRTEM image (b) of CE/ $\mathrm{SnS}_{2}$ composites. 

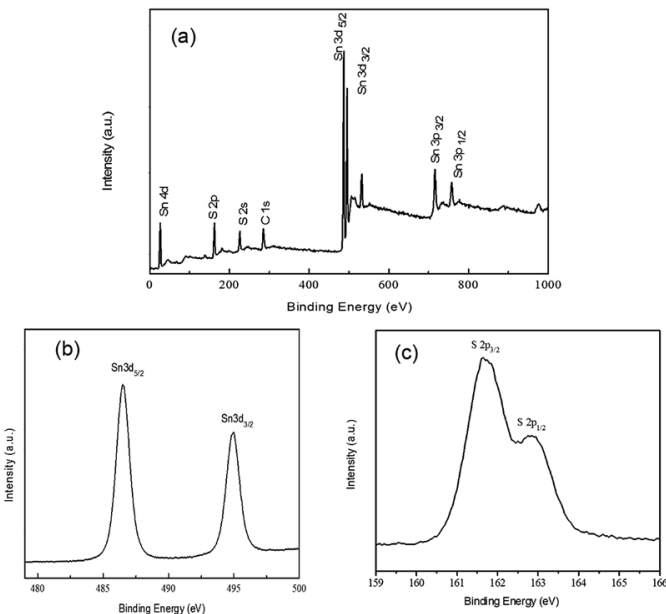

Fig. 13 XPS spectra of the CE/SnS 2 composites: (a) survey; (b) Sn 3d; (c) $S 2 p$.

of pure $\mathrm{SnS}_{2}$, and it is also consistent with the result of EDS shown in Fig. 11.

The results of BET analysis of $\mathrm{CE} / \mathrm{SnS}_{2}$ composite with the nitrogen adsorption-desorption isotherm are shown in Fig. 14. The well-defined steps of the isotherm illustrate the mesoporous structure of $\mathrm{CE} / \mathrm{SnS}_{2}$ composite. The BET surface area of $\mathrm{CE} / \mathrm{SnS}_{2}$ composite was measured to be $23.1462 \mathrm{~m}^{2} \mathrm{~g}^{-1}$, which is larger than the value of pristine $\mathrm{SnS}_{2}$ nanoflowers $\left(14.4730 \mathrm{~m}^{2}\right.$ $\mathrm{g}^{-1}$, shown in Table 1). Both pristine $\mathrm{SnS}_{2}$ and $\mathrm{CE} / \mathrm{SnS}_{2}$ composite exhibit a multi-modal mesoporous nature (BJH analysis, the inset of Fig. 14). The small pores presumably arise from the nanopetals, whereas the large pores may be attributed to the inter-nanopetals spaces. Compared with the pristine $\mathrm{SnS}_{2}$, the enhanced specific surface area of the composite can enlarge the inner space for absorption or desorption of degradation pollutant, which enhance greatly photocatalytic efficiency of the composite.

3.2.2 Effect of synthesis conditions. Influence of preparation conditions on the formation of $\mathrm{CE} / \mathrm{SnS} \mathrm{S}_{2}$ composite was investigated by a series of comparative experiments carried

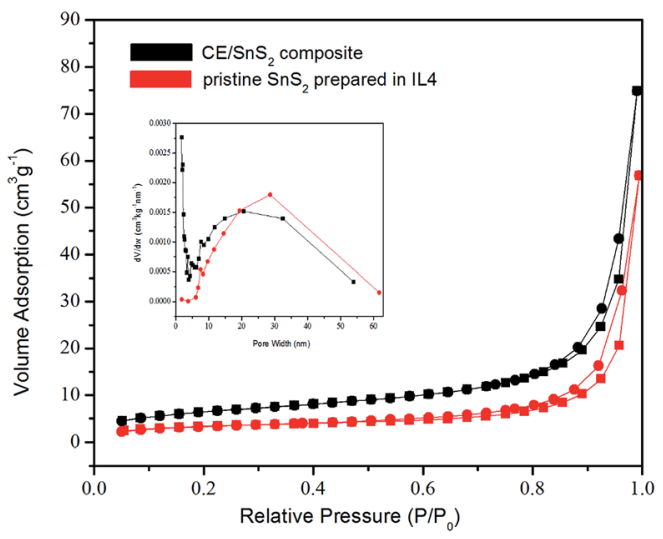

Fig. $14 \mathrm{~N}_{2}$ adsorption-desorption isotherm for the CE/SnS 2 composites. out by varying the experimental parameters, such as microwave irradiation time and reaction temperature. Considering the easy thermal degradation of CE at high temperature, the microwave irradiation temperature did not exceed $120^{\circ} \mathrm{C}$. As shown in Fig. 15a, the $40 \mathrm{~min}$ and $80^{\circ} \mathrm{C}$ is not sufficient for effective $\mathrm{SnS}_{2}$ nucleation and crystal growth, no peaks corresponding to the existence of $\mathrm{SnS}_{2}$ are detected. Under this condition nothing is observed on the surface of CE (Fig. 13b). Increasing of irradiation temperature promotes $\mathrm{SnS}_{2}$ particles formation $\left(\geq 100{ }^{\circ} \mathrm{C}\right.$ ), which can be verified by the detection of 2D nanosheets on the CE surface (Fig. 15b) and the presence of XRD peaks assigned to crystalline $\mathrm{SnS}_{2}$ (Fig. 15a). Well-developed flower-like $\mathrm{SnS}_{2}$ nanosheets is obtained at $120{ }^{\circ} \mathrm{C}$ when reaction time was $\geq 80 \mathrm{~min}$ (Fig. 15b). However, when the irradiation temperature is set at $140{ }^{\circ} \mathrm{C}$, the peak assigned to $\mathrm{CE}$ at $2 \theta=21.32^{\circ}$ became weaker (Fig. 15a), showing decomposition of CE occurred at $140{ }^{\circ} \mathrm{C}$. Therefore, the reaction temperature of $100-120^{\circ} \mathrm{C}$ and reaction time no less than $80 \mathrm{~min}$ would be suitable for the synthesis of $\mathrm{CE} / \mathrm{SnS}_{2}$.

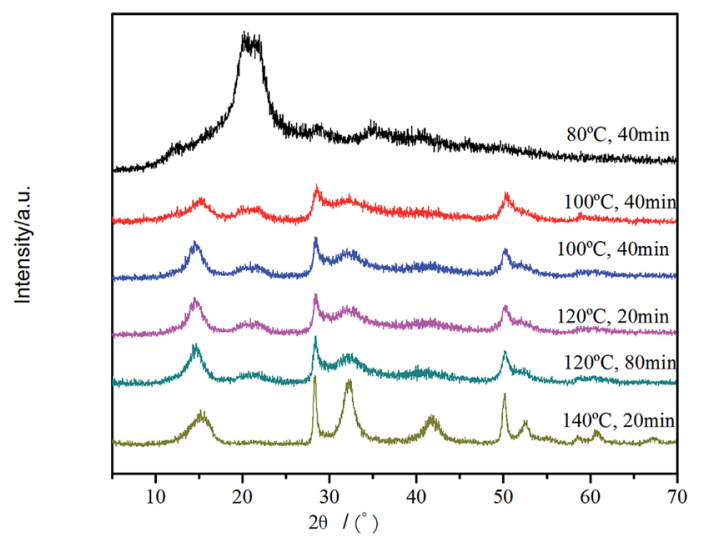

(a)

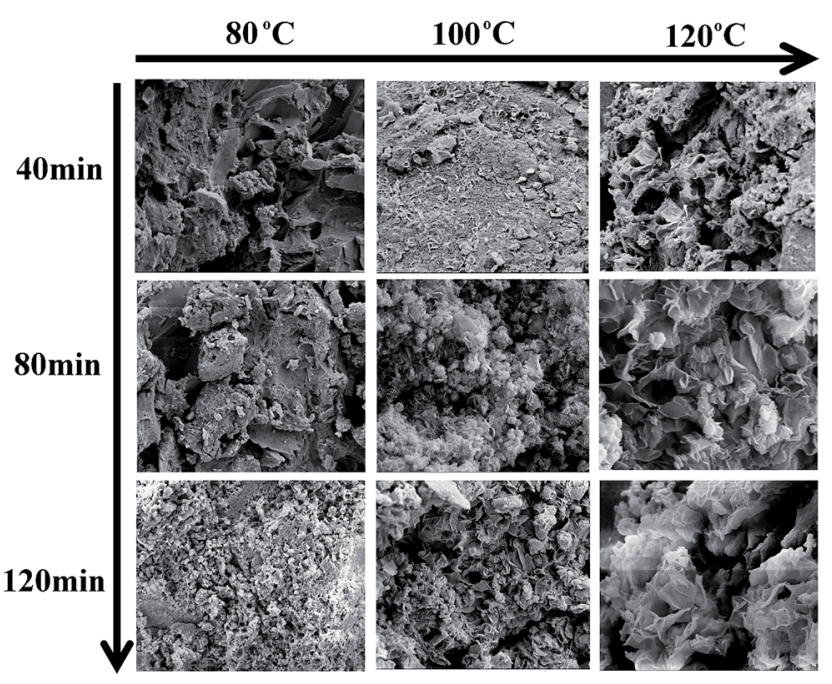

(b)

Fig. 15 XRD patterns (a) and SEM images (b) of composite prepared in different conditions. 


\subsection{Photocatalytic performance of the $\mathrm{CE} / \mathrm{SnS}_{2}$ composite}

The photocatalytic degradation of rhodamine B (RhB) under visible light irradiation was used to evaluate the photocatalytic performances of $\mathrm{CE} / \mathrm{SnS}_{2}$ composite. As a comparison, pristine $\mathrm{SnS}_{2}$ or CE was also tested under identical conditions. The blank test shows that RhB cannot be degraded under visible light irradiation without catalysts, indicating that the RhB are stable molecules and the photolysis can be ignored. It can be seen from the Fig. 15 that the adsorption-desorption equilibrium of RhB on the catalyst or cellulose surfaces have been almost achieved in $60 \mathrm{~min}$ before visible-light irradiation. The influence of pollutant adsorption on the photocatalysts can be largely neglected in the photocatalytic process by using the pollutant concentration after the adsorption-desorption equilibrium as the original concentration $\left(C_{0}\right)$ for the degradation rates measurements.

As shown in Fig. 16, almost no RhB degradation is observed in the presence of $\mathrm{CE}$. In comparison, both of adsorptive ratios and degradation rates are remarkably enhanced when pristine $\mathrm{SnS}_{2}$ and $\mathrm{CE} / \mathrm{SnS}_{2}$ are used as the photocatalysts. The degradation rate of $\mathrm{RhB}$ is $66.2 \%$ after $300 \mathrm{~min}$ irradiation, while this value reached almost $100 \%$ for the $\mathrm{CE} / \mathrm{SnS}_{2}$ composite after $150 \mathrm{~min}$. The results indicate clearly that the introduction of $\mathrm{CE}$ can enhance the photocatalytic performance of $\mathrm{SnS}_{2}$.

To find out the reason for enhanced visible light photocatalytic activity of $\mathrm{CE} / \mathrm{SnS}_{2}$ composite, the UV-vis DRS was used to determine the band gap energy of the synthesized samples (Fig. 17). Compared to pristine $\mathrm{SnS}_{2}, \mathrm{CE} / \mathrm{SnS}_{2}$ composite shows an increased optical absorbance in both UV and visible range. The composite can allow multiple scattering of UV-vis light, suggesting that the optical path length for light transporting through those $\mathrm{CE} / \mathrm{SnS}_{2}$ composite structures might be longer than that for pristine $\mathrm{SnS}_{2}$. The longer optical path length can increase the quantity of photogenerated electrons and holes available to participate in the photocatalytic decomposition of the contaminants. The band gap of pristine $\mathrm{SnS}_{2}$ was evaluated to be $1.80 \mathrm{eV}$, whereas the band-gap of $\mathrm{CE} / \mathrm{SnS}_{2}$ composite was reduced to $1.69 \mathrm{eV}$, which could be attributed to the chemical bonding between $\mathrm{SnS}_{2}$ and the specific sites of CE. The narrower band gap of $\mathrm{CE} / \mathrm{SnS}_{2}$ composite indicates the enhanced ability to absorb visible light, which is beneficial to the photocatalytic performance.

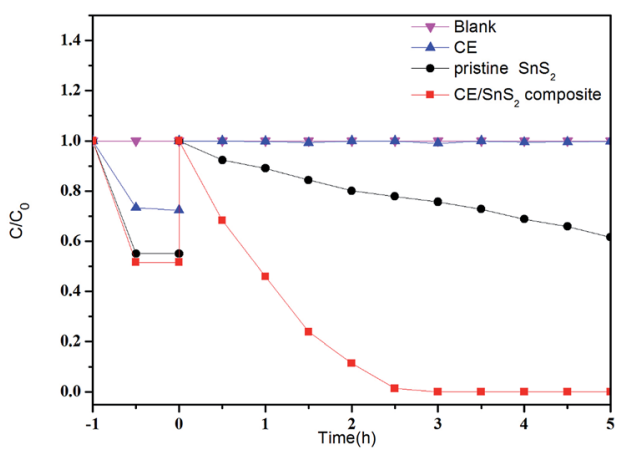

Fig. 16 Photocatalytic degradation of $\mathrm{RhB}$ by $\mathrm{CE} / \mathrm{SnS}_{2}$ composite under visible-light irradiation.

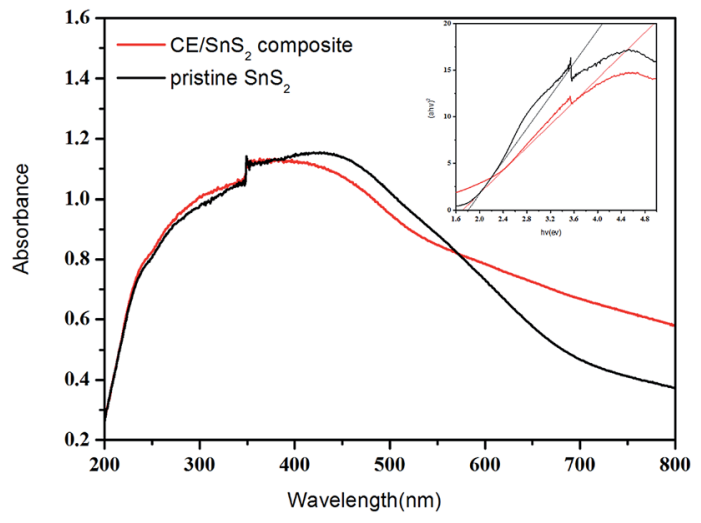

Fig. 17 UV-vis solid absorption spectra and the corresponding band gap values (inset) of $\mathrm{CE} / \mathrm{SnS}_{2}$ composites.

The migration, transfer and recombination processes of photo-generated electron-hole pairs in $\mathrm{CE} / \mathrm{SnS}_{2}$ composite was investigated via photoluminescence (PL) technique. As shown in Fig. 18, pristine $\mathrm{SnS}_{2}$ has a strong, wide peak in the PL spectrum excited at $365 \mathrm{~nm}$, while the $\mathrm{PL}$ peak of the $\mathrm{CE} / \mathrm{SnS}_{2}$ composite decreases remarkably, which indicates that the recombination of electrons and holes is hindered greatly. Photocurrents were measured for pristine $\mathrm{SnS}_{2}$ and $\mathrm{CE} / \mathrm{SnS}_{2}$ composite to investigate the transmission of photogenerated carriers (Fig. 19). Under visible light irradiation, the

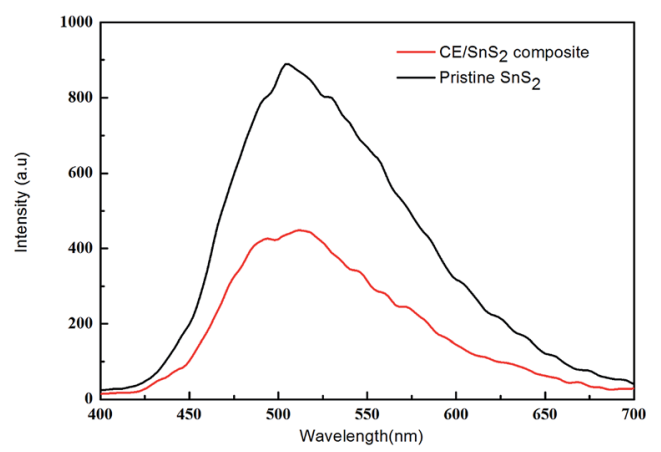

Fig. 18 Photoluminescence (PL) spectra under visible light of pristine $\mathrm{SnS}_{2}$ and $\mathrm{CE} / \mathrm{SnS}_{2}$.

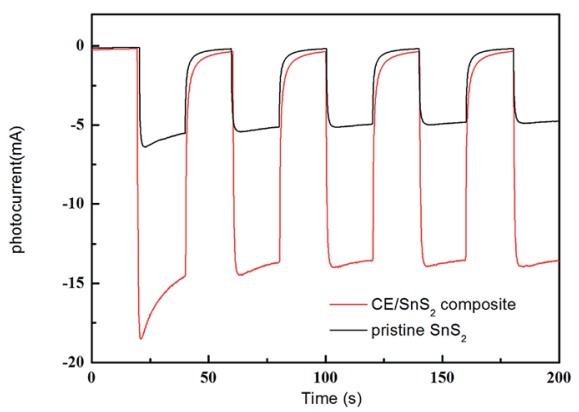

Fig. 19 Transient photocurrent responses of pristine $\mathrm{SnS}_{2}$ and CE/ $\mathrm{SnS}_{2}$ composite under visible light. 


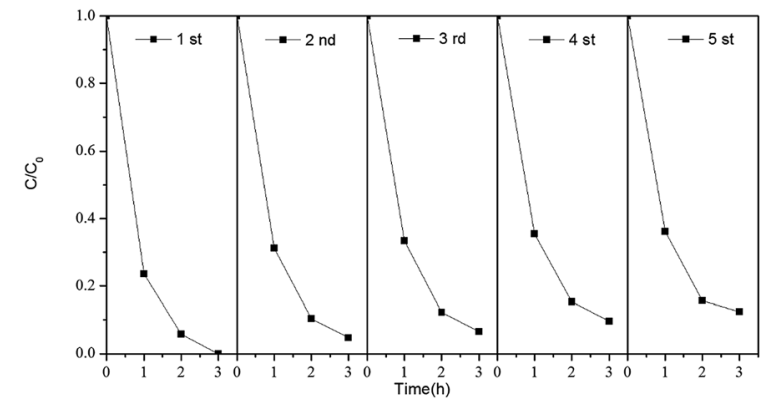

Fig. 20 Cycling runs for the photocatalytic degradation of RhB by CE/ $\mathrm{SnS}_{2}$ composite under visible light irradiation.

photocurrents of $\mathrm{CE} / \mathrm{SnS}_{2}$ composite are much higher than that of pristine $\mathrm{SnS}_{2}$, indicating the enhanced separation and transition of electrons and holes. Moreover, it is generally accepted that the catalytic process is related to the adsorption of reactant molecules on the surface of catalyst. ${ }^{43,44}$ The larger specific surface area of composite can result in a more unsaturated surface coordination sites exposed to the solution and enlarge the absorption of reactant molecules, thus enhancing the photocatalytic efficiency. Additionally, the high surface-tovolume ratios of the $\mathrm{CE} / \mathrm{SnS}_{2}$ composite are in favor of the transfer of electrons and holes ${ }^{\mathbf{4 5 , 4 6}}$ and facilitate the degradation of pollution molecules.

\subsection{Stability of the $\mathrm{CE} / \mathrm{SnS}_{2}$ composite}

The stability and reusability of photocatalysts is another important issue for their practical applications. Therefore, the circulating runs in the photocatalytic degradation of RhB in the presence of $\mathrm{SnS}_{2}$ under visible-light were checked. In this work, $\mathrm{SnS}_{2}$ was recycled for four times in the same photocatalytic reactions. After each reuse cycle which lasted for $3 \mathrm{~h}$, the composite was separated from the aqueous suspension by filtration, washed with deionized water and ethanol, dried, and weighed for the next reuse cycle. Taking into account the mass loss of composite during each reuse cycle, the former reuse cycle must be conducted twice in order to accumulate enough samples for the latter reuse cycle. Fig. 20 shows the photocatalytic performance of $\mathrm{CE} / \mathrm{SnS}_{2}$ composite in the first four reuse cycles. Obviously, the photocatalytic activities of $\mathrm{CE} / \mathrm{SnS}_{2}$ composite deteriorated gradually with the increase in the number of reuse cycles. Nevertheless, even in the fourth reuse cycle of $\mathrm{CE} / \mathrm{SnS}_{2}$, the degradation ratio of $\mathrm{RhB}$ can still reach $87.6 \%$ upon visible light irradiation for $180 \mathrm{~min}$.

\section{Conclusions}

In summary, a novel $\mathrm{CE} / \mathrm{SnS}_{2}$ composite was successfully synthesized by a one-step microwave-assisted ionic liquid method using $\mathrm{SnCl}_{4} \cdot 5 \mathrm{H}_{2} \mathrm{O}$, thioacetamide and $\mathrm{CE}$ as starting materials. The proposed method has the advantages of fast and simple process. The morphology variations of the assynthesized $\mathrm{SnS}_{2}$ were achieved by simply varying the types of ILs in the system. The obtained $\mathrm{CE} / \mathrm{SnS}_{2}$ composite exhibits higher photocatalytic activity than that of pristine $\mathrm{SnS}_{2}$ on the degradation of $\mathrm{RhB}$ under visible light irradiation. The enhanced photocatalytic activities of $\mathrm{CE} / \mathrm{SnS}_{2}$ composite can be ascribed to their increase optical absorbance in the available light energy region, higher BET surface area and efficient rate of separation and transporting of the photogenerated electrons and holes in the $\mathrm{CE} / \mathrm{SnS}_{2}$ composite. The resulting high visible light-driven photocatalytic activity and good stability of $\mathrm{CE} / \mathrm{SnS}_{2}$ composite are very promising photocatalysts for degrading organic pollutants and the present method may open up the opportunity to design and synthesize other novel semiconductor materials with special properties.

\section{Acknowledgements}

This research was supported by National Natural Science Foundation of China (No. 21577018, No. 2140070158), the Science and Technology Project of Fujian Province Educational Department (No. JZ160416), Natural Science Foundation of Fujian Province, China (No. 2015J01049), the Independent Research Project of State Key Laboratory of Photocatalysis on Energy and Environment, China (No. 2014C02).

\section{References}

1 H. Choi, A. C. Sofranko and D. D. Dionysiou, Adv. Funct. Mater., 2006, 16, 1067-1074.

2 M. D. Hernandez-Alonso, F. Fresno, S. Suarez and J. M. Coronado, Energy Environ. Sci., 2009, 2, 1231-1257.

3 S. G. Kumar and L. G. Devi, J. Phys. Chem. A, 2011, 115, 13211-13241.

4 H. Liu, W. R. Cao, Y. Su, Y. Wang and X. H. Wang, Appl. Catal., B, 2012, 111-112, 271-279.

5 Y. C. Zhang, Z. N. Du, S. Y. Li and M. Zhang, Appl. Catal., B, 2010, 95, 153-159.

6 J. Li, T. Wang and X. Du, Sep. Purif. Technol., 2012, 101, 1117.

7 R. Lucena, F. Fresno and J. C. Conesa, Appl. Catal., A, 2012, 415, 111-117.

8 J. T. Zai, X. F. Qian, K. X. Wang, C. Yu, L. Q. Tao, Y. L. Xiao and J. S. Chen, CrystEngComm, 2012, 14, 5627-5633.

9 H. Tong, Y. J. Zhu, L. X. Yang, L. Li and L. Zhang, Angew. Chem., Int. Ed., 2006, 45, 7739-7742.

10 C. Pacholski, A. Kornowski and H. Weller, Angew. Chem., Int. Ed., 2002, 41, 1188-1191.

11 Z. Y. Tang, N. A. Kotow and M. Giersig, Science, 2002, 297, 237-240.

12 A. Kudo and S. Hijii, Chem. Lett., 1999, 10, 1103-1104.

13 H. S. Kim, Y. H. Chung, S. H. Kang and Y. E. Sung, Electrochim. Acta, 2009, 54, 3606-3610.

14 X. Liu and H. Bai, Powder Technol., 2013, 237, 610-615.

15 P. Balaz, T. Ohtani, Z. Bastl and E. Boldizarova, J. Alloys Compd., 2002, 337, 76-82.

16 S. Y. Hong, R. Popovitz-Biro, Y. Prior and R. Tenne, J. Am. Chem. Soc., 2003, 125, 10470-10474.

17 A. Yella, E. Mugnaioli, H. A. Therese, M. Panthofer, U. Kolb and W. Tremel, Angew. Chem., Int. Ed., 2009, 48, 6426-6430. 
18 T. T. Huong, L. T. Vinh, H. T. Phuong, H. T. Khuyen, T. K. Anh, V. D. Tu and L. Q. Minh, J. Lumin., 2016, 173, 89-93.

19 H. T. Hu, X. B. Wang, F. M. Liu, J. C. Wang and C. H. Xu, Synth. Met., 2011, 161, 404-410.

20 Y. Chen, W. Li, J. Y. Wang, Y. Gan, L. Liu and M. Ju, Appl. Catal., B, 2016, 191, 94-105.

21 P. Giesz, G. Celichowski, D. Puchowicz, I. Kaminska, J. Grobelny, D. Batory and M. Cieslak, Cellulose, 2016, 23, 2143-2159.

22 D. W. Synnott, M. K. Seery, S. J. Hinder, J. Colreavy and S. C. Pillai, Nanotechnology, 2013, 24, 045704.

23 J. A. Dahl, B. L. S. Maddux and J. E. Hutchison, Chem. Rev., 2007, 107, 2228-2269.

24 M. Antonietti, D. Kuang, B. Smarsly and Y. Zhou, Angew. Chem., Int. Ed., 2004, 43, 4988-4992.

25 K. L. Ding, Z. J. Miao, Z. M. Liu, Z. F. Zhang, B. X. Han, G. M. An, S. D. Miao and Y. Xie, J. Am. Chem. Soc., 2007, 129, 6362-6363.

26 E. Kowsari and G. Faraghi, Mater. Res. Bull., 2010, 45, 939. 27 W. W. Wang and Y. J. Zhu, Cryst. Growth Des., 2005, 5, 505. 28 M. Sabbaghan, J. Beheshtian and S. A. M. Mirsaeidi, Ceram. Int., 2014, 40, 7769-7774.

29 A. Taubert and Z. Li, Dalton Trans., 2007, 7, 723-727.

30 Y. Chen, W. Z. Li, J. Y. Wang, Y. L. Gan, L. Liu and M. T. Ju, Appl. Catal., B, 2016, 191, 94-105.

31 J. Di, J. X. Xia, S. Yin, H. Xu, M. Q. He, H. M. Li, L. Xu and Y. P. Jiang, RSC Adv., 2013, 3, 19624-19631.
32 H. Zhang, J. Wu, J. Zhang and J. He, Macromolecules, 2005, $38,8272$.

33 D. V. Bavykin, V. N. Parmon, A. A. Lapkin and F. C. Walsh, J. Mater. Chem., 2004, 14, 3370-3377.

34 U. G. Akpan and B. H. Hameed, J. Hazard. Mater., 2009, 170, 520-529.

35 C. Chang, L. Y. Zhu, Y. Fu and X. L. Chu, Chem. Eng. J., 2013, 233, 305-314.

36 G. Cheng, J. Y. Xiong, F. J. S. Tadler and J. Florian, New J. Chem., 2013, 37, 3207-3213.

37 S. X. Weng, B. B. Chen, L. Y. Xie, Z. Y. Zheng and P. Liu, J. Mater. Chem. A, 2013, 1, 3068-3075.

38 Y. Jiang and Y. J. Zhu, J. Phys. Chem. B, 2005, 109, 4361-4364. 39 X. Liu, J. Ma and W. Zheng, Rev. Adv. Mater. Sci., 2011, 27, 43-51.

40 C. A. Formstone, E. T. Fitzgerald, P. A. Cox and D. Ohare, Inorg. Chem., 1990, 29, 3860-3866.

41 Y. C. Zhang, Z. N. Du, K. W. Li and M. Zhang, Sep. Purif. Technol., 2011, 81, 101-107.

42 Y. Lei, S. Song, W. Fan, Y. Xing and H. Zhang, J. Phys. Chem. C, 2009, 113, 1280-1285.

43 D. Ma, S. Huang, W. Chen, S. Hu, F. Shi and K. Fan, J. Phys. Chem. C, 2009, 113, 4369-4374.

44 X. Xiao and W. D. Zhang, RSC Adv., 2011, 1, 1099-1105.

45 L. P. Zhu, G. H. Liao, N. C. Bing, L. L. Wang, Y. Yang and H. Y. Xie, CrystEngComm, 2010, 12, 3791-3796.

46 X. Xiao and W. D. Zhang, J. Mater. Chem., 2010, 20, 58665870. 